2010-01-01

\title{
Study of the Effect of Source Signal Bandwidth on Ratiometric Wavelength Measurement
}

\author{
Qiang wu \\ Technological University Dublin, qiang.wu@tudublin.ie \\ Yuliya Semenova \\ Technological University Dublin, yuliya.semenova@tudublin.ie \\ Ginu Rajan \\ Technological University Dublin, ginu.rajan@tudublin.ie
}

See next page for additional authors

Follow this and additional works at: https://arrow.tudublin.ie/engscheceart

Part of the Electrical and Computer Engineering Commons

\section{Recommended Citation}

Wu, Q. et al. (2010) Study of the Effect of Source Signal Bandwidth on Ratiometric Wavelength

Measurement. Applied Optics, Vol.49, no.29, pp.5626. doi: 10.1364/A0.49.005626

This Article is brought to you for free and open access by the School of Electrical and Electronic Engineering at ARROW@TU Dublin. It has been accepted for inclusion in Articles by an authorized administrator of ARROW@TU Dublin. For more information, please contact arrow.admin@tudublin.ie, aisling.coyne@tudublin.ie, gerard.connolly@tudublin.ie.

Funder: Science Foundation Ireland under grant 07/SK/

11200.

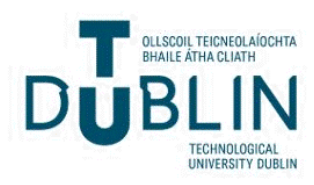


Authors

Qiang wu, Yuliya Semenova, Ginu Rajan, Pengfei Wang, and Gerald Farrell

This article is available at ARROW@TU Dublin: https://arrow.tudublin.ie/engscheceart/91 


\title{
Study of the effect of source signal bandwidth on ratiometric wavelength measurement
}

\author{
Qiang Wu, ${ }^{*}$ Yuliya Semenova, Ginu Rajan, Pengfei Wang, and Gerald Farrell \\ Photonics Research Centre, School of Electronic and Communications Engineering, \\ Dublin Institute of Technology, Kevin Street, Dublin 8, Ireland \\ ${ }^{*}$ Corresponding author: qiang.wu@ dit.ie
}

Received 6 July 2010; revised 9 September 2010; accepted 9 September 2010; posted 10 September 2010 (Doc. ID 131088); published 7 October 2010

\begin{abstract}
We derive an analytic equation for a ratiometric wavelength measurement system and analyze the influence of the optical source signal bandwidth. Our investigation shows that in a particular optical sensing system, the higher the bandwidth of the optical signal, the better resolution the system will achieve. Experiments based on two types of optical signals (output signal of a tunable laser and a fiber Bragg grating) were carried out, and experimental results verified both the simulation results and the theoretical analysis. (C) 2010 Optical Society of America
\end{abstract}

OCIS codes: $\quad 060.2370,060.3735$.

\section{Introduction}

Wavelength measurements are very important in many optical systems, such as dense wavelength division multiplexing optical communication systems and sensing systems [1-7]. Ratiometric wavelength measurement (RWM) is a simple, high-speed, and cost effective technique compared to wavelength scanning based active techniques [8-13]. Previous investigations on RWM systems were based only on numerical simulations without an analytical formulation, which cannot provide a straightforward analysis for the ratiometric system $[12,13]$. Moreover, these investigations on the impact of the nature of the input optical signal have been limited to SNR, but without considering the impact of optical source signal bandwidth, which influences the ratio response of the ratiometric wavelength system and hence the resolution of the measurement.

This paper derives an analytical equation for the ratio response in a RWM system and provides an analysis of the effect of an optical signal bandwidth on the ratio response based on the analytical equation. This analysis is verified by both simulation

$0003-6935 / 10 / 295626-06 \$ 15.00 / 0$

(C) 2010 Optical Society of America and experimental results. In Section 2, a theoretical analysis of the ratio response is presented and an analytical equation for the ratiometric system is provided based on a rectangular optical signal. In Section 3, numerical simulations were carried out to verify the theoretical analysis. Experimental verification is also reported using two types of optical source signals: the signal from a tunable laser and the signal reflected from the fiber Bragg grating (FBG).

\section{Theoretical Analysis}

A RWM system normally comprises two arms: one is a reference arm; the other is a signal arm containing an edge filter. The schematic diagram is shown in Fig. 1.

Two photodiodes are placed at the ends of each arm to measure the optical power and, hence, the power ratio of the system. In such a system, assuming the splitter is wavelength independent and provides an equal input to the two arms, the ratio of the outputs of the two photodiodes at a wavelength $\lambda_{0}$ can be expressed as

$$
R\left(\lambda_{0}\right)=-10 \log _{10}\left[\frac{\int I_{\lambda_{0}}(\lambda) T(\lambda) \mathrm{d} \lambda+N_{e}}{\int I_{\lambda_{0}}(\lambda) \mathrm{d} \lambda+N_{r}}\right],
$$




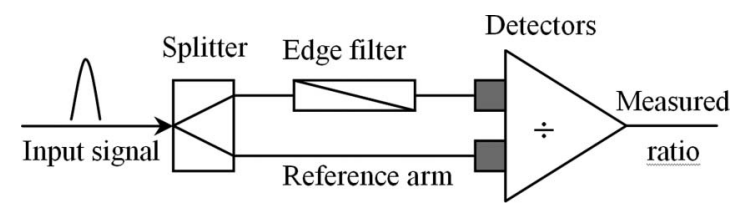

Fig. 1. Schematic diagram of a ratiometric wavelength measurement system.

where $I_{\lambda_{0}}(\lambda)$ is the input power spectral density and $T(\lambda)$ is the transmission response of the edge filter. In this formula, to represent the electrical noise resulting from optoelectronic conversion, an equivalent optical signal level is used, which has both a fixed component and a random component, to represent both the photodiode dark current and shot noise, respectively. The terms $N_{e}$ and $N_{r}$ represent the equivalent noise of the photodiodes connected to the edge filter and reference signal arms, respectively.

A simple case for the transmission response of the edge filter, $T(\lambda)$, in the wavelength range from $\lambda_{10}$ to $\lambda_{20}$ is a linear function as expressed below:

$$
\begin{aligned}
\bar{T}(\lambda) & =\bar{T}\left(\lambda_{10}\right)+\frac{\bar{T}\left(\lambda_{20}\right)-\bar{T}\left(\lambda_{10}\right)}{\lambda_{20}-\lambda_{10}}\left(\lambda-\lambda_{10}\right) \\
& =\bar{T}\left(\lambda_{10}\right)+m\left(\lambda-\lambda_{10}\right),
\end{aligned}
$$

where $m$ is the slope of the edge filter, and

$$
\bar{T}(\lambda)=-10 \log _{10}[T(\lambda)] .
$$

In practice, the input signal has a limited signalto-noise ratio (SNR). The simplest case is to assume that the input optical signal has a rectangular spectrum, which can be expressed as

$$
\begin{gathered}
I_{\lambda_{0}}(\lambda)=\left\{\begin{array}{ccc}
P & \left|\lambda-\lambda_{0}\right| \leq \Delta \lambda_{0} / 2 & \lambda_{1} \leq \lambda \leq \lambda_{2} \\
N_{s} & \left|\lambda-\lambda_{0}\right|>\Delta \lambda_{0} / 2 & \lambda_{1} \leq \lambda \leq \lambda_{2}
\end{array}\right. \\
\mathrm{SNR}=-10 \log _{10}\left(\frac{N_{s}}{P}\right),
\end{gathered}
$$

where $P$ is the signal peak power spectral density, $N_{s}$ is the noise power spectral density of the optical signal in a wavelength range $\left(\lambda_{1} \leq \lambda \leq \lambda_{2}\right)$, and $\Delta \lambda_{0}$ is the bandwidth of the rectangular signal spectrum. Substituting Eq. (4) in Eq. (1), we have
Substituting Eqs. (2) and (3) into Eq. (6), we have Eq. (7):

$$
\begin{aligned}
R\left(\lambda_{0}\right)= & -10 \log _{10}\left\{\frac{T\left(\lambda_{0}\right)\left[P A-N_{s} A+N_{s} B+N_{e} / T\left(\lambda_{0}\right)\right]}{P C}\right\} \\
& =\bar{T}\left(\lambda_{0}\right)-10 \log _{10}\left[A-\frac{N_{s}}{P} A+\frac{N_{s}}{P} B+\frac{N_{e}}{P T\left(\lambda_{0}\right)}\right] \\
& +10 \log _{10}(C)
\end{aligned}
$$

where

$$
\begin{gathered}
A=\frac{10\left(10^{m \Delta \lambda_{0} / 20}-10^{-m \Delta \lambda_{0} / 20}\right)}{m \ln 10}, \\
B=\frac{10\left(10^{-m \lambda_{1} / 10}-10^{-m \lambda_{2} / 10}\right)}{m \ln 10} 10^{m \lambda_{0} / 10}, \\
C=\Delta \lambda_{0}+\frac{N_{s}\left(\lambda_{2}-\lambda_{1}-\Delta \lambda_{0}\right)}{P}+\frac{N_{r}}{P} .
\end{gathered}
$$

We can assume that in a ratiometric system the minimum reliably detectable ratio variation is limited by the peak-to-peak ratio fluctuations $\Delta R_{p-p}$ induced by noise. If the slope of the ratio response of a system is $S\left(\lambda_{0}\right)=d R\left(\lambda_{0}\right) / d \lambda_{0}$, then the system resolution is equal to

$$
D\left(\lambda_{0}\right)=\frac{\Delta R_{p-p}}{S\left(\lambda_{0}\right)}
$$

Based on the equations above, three cases were studied:

1. Both photodiode and optical source are noise free.

In an ideal situation (the SNR of the signal is very high, and the photodiodes are noise free), the ratio response of the wavelength measurement system in Eq. (7) can be simplified as

$$
R\left(\lambda_{0}\right)=\bar{T}\left(\lambda_{0}\right)-10 \log _{10}(A)+10 \log _{10}\left(\Delta \lambda_{0}\right) .
$$

Equation (12) shows that the slope of the ratio $R\left(\lambda_{0}\right)$ is identical to the slope of the transmission response of the edge filter $\bar{T}\left(\lambda_{0}\right)$. There is a difference

$$
R\left(\lambda_{0}\right)=-10 \log _{10}\left\{\frac{P \int_{\lambda_{0}-\Delta \lambda_{0} / 2}^{\lambda_{0}+\Delta \lambda_{0} / 2} T(\lambda) \mathrm{d} \lambda+N_{s}\left[\int_{\lambda_{1}}^{\lambda_{0}-\Delta \lambda_{0} / 2} T(\lambda) \mathrm{d} \lambda+\int_{\lambda_{0}+\Delta \lambda_{0} / 2}^{\lambda_{2}} T(\lambda) \mathrm{d} \lambda\right]+N_{e}}{P \Delta \lambda_{0}+N_{s}\left(\lambda_{2}-\lambda_{1}-\Delta \lambda_{0}\right)+N_{r}}\right\} .
$$


term $-10 \log _{10}(A)+10 \log _{10}\left(\Delta \lambda_{0}\right)$, but for a particular optical sensing system, where the bandwidth $\Delta \lambda_{0}$ is known, the difference term $-10 \log _{10}(A)+$ $10 \log _{10}\left(\Delta \lambda_{0}\right)$ is a constant value and, therefore, can be calibrated out in the system. In this case the system ratio is a simple function of the slope of the edge filter. From Eqs. (2) and (11), it is easy to determine that the higher the slope of the edge filter, the higher the value of $S\left(\lambda_{0}\right)$, and the higher the resolution of the system.

2. Photodiode is noise free but SNR is limited.

If the photodiodes are noise free but the noise level of the optical signal is significant, the ratio function and slope of the ratio response can be expressed as follows:

$$
\begin{aligned}
R\left(\lambda_{0}\right)= & \bar{T}\left(\lambda_{0}\right)-10 \log _{10}\left(A-\frac{N_{s}}{P} A+\frac{N_{s}}{P} B\right) \\
& +10 \log _{10}(C) \\
S\left(\lambda_{0}\right)=m-\frac{N_{s} B / P}{A-N_{s} A / P+N_{s} B / P} & m .
\end{aligned}
$$

To simplify the analysis we assume that $m$ is a positive number. From Eqs. (8) and (9) it can be seen that the second term in Eq. (14) is a function of wavelength. At shorter wavelengths, the value of $N_{s} B / P$ is much smaller than that of $A$. In this case, the slope $S\left(\lambda_{0}\right)$ is approximately equal to $m$. However, factor $B$ increases sharply as the wavelength $\lambda_{0}$ moves toward longer wavelengths. As the wavelength increases and $N_{s} B / P$ is comparable to that of $A$, the term

$$
\frac{N_{s} B / P}{A-N_{s} A / P+N_{s} B / P} m
$$

can no longer be neglected. In this case as the wavelength increases, the slope $S\left(\lambda_{0}\right)$ decreases and, hence, the resolution of the system decreases. It also can be shown from Eq. (14) that for a given SNR, the higher the bandwidth $\overline{\Delta \lambda}_{0}$, the larger the value $A$, and, hence, the smaller the value

$$
\frac{N_{s} B / P}{A-N_{s} A / P+N_{s} B / P} m
$$

and, hence, the higher the slope $S\left(\lambda_{0}\right)$. This indicates that for an optical source signal with the same SNR but a higher bandwidth, the effective slope $S\left(\lambda_{0}\right)$, and, hence, the resolution of the measurement system, will be higher.

3. Noise of both photodiode and optical signal is significant.

If the noise of both the photodiodes and the optical signal is significant, the slope of the ratio of the system is

$$
\begin{aligned}
& S\left(\lambda_{0}\right)=m-\frac{1}{A-\frac{N_{s}}{P} A+\frac{N_{s}}{P} B+\frac{N_{e}}{P T\left(\lambda_{0}\right)}} \\
& \quad \times\left(\frac{N_{s}}{P} B+\frac{N_{e}}{P} 10^{\frac{\bar{T}\left(\lambda_{0}\right)}{10}}\right) m .
\end{aligned}
$$

Because the noise of both the photodiodes and the optical signal is significant, both $B$ and $\frac{N_{e}}{P T\left(\lambda_{0}\right)}$ are influenced by the wavelength. At shorter wavelengths, both $N_{s} B / P$ and $\frac{N_{e}}{P} 10^{\frac{T\left(\lambda_{0}\right)}{10}}$ are very small compared to $A$. The term

$$
\frac{1}{A-\frac{N_{s}}{P} A+\frac{N_{s}}{P} B+\frac{N_{e}}{P T\left(\lambda_{0}\right)}}\left(\frac{N_{s}}{P} B+\frac{N_{e}}{P} 10^{\frac{T\left(\lambda_{0}\right)}{10}}\right) m
$$

can thus be treated as zero and, hence, the slope $S\left(\lambda_{0}\right)$ is approximately equal to $m$. As the wavelength increases, the values of both $N_{s} B / P$ and $\frac{N_{e}}{P} 10^{\frac{T}{\frac{T}{\left(\lambda_{0}\right)}}}$ increase dramatically. If the value of either $N_{s} B / P$ or $\frac{N_{e}}{P T\left(\lambda_{0}\right)}$ is comparable to $A$, the term

$$
\frac{1}{A-\frac{N_{s}}{P} A+\frac{N_{s}}{P} B+\frac{N_{e}}{P T\left(\lambda_{0}\right)}}\left(\frac{N_{s}}{P} B+\frac{N_{e}}{P} 10^{\frac{\bar{T}\left(\lambda_{0}\right)}{10}}\right) m
$$

cannot be neglected. In this case, as the wavelength increases, the slope $S\left(\lambda_{0}\right)$ decreases and, hence, the resolution of the system decreases. A consequence is that optical sources that have a larger bandwidth will suffer less from resolution degradation because, as shown in Eq. (15), a larger bandwidth will mean a

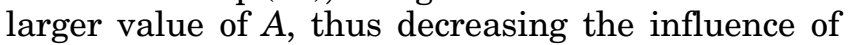
$N_{s} B / P$ or $\frac{N_{e}}{P T\left(\lambda_{0}\right)}$.

\section{Numerical Simulations}

The analysis above was based on a rectangular signal spectrum, and it gives us a simple but effective insight into the influence of the optical signal bandwidth $\Delta \lambda_{0}$ on the $R\left(\lambda_{0}\right)$ and $S\left(\lambda_{0}\right)$ in a real ratiometric system. In practice, optical signals rarely have a rectangular power spectral density, and it must be determined whether the analysis based on a rectangular signal spectrum is suitable for a real optical signal spectrum such as a Gaussian signal spectrum. In order to do so, simulation investigations were carried out using the original Eq. (1).

As illustrated in [14], the laser line shape changes from Lorentzian to Gaussian in a continuous way as the correlation time of the frequency fluctuations increases from zero to infinity. In our case, we deal with the output spectral response of a tunable laser as a Gaussian source signal

$$
I_{\lambda_{0}}(\lambda)=\left\{\begin{array}{ccc}
P \exp \left[-4 \ln 2\left(\frac{\lambda-\lambda_{0}}{\Delta \lambda_{0}}\right)^{2}\right] & \left|\lambda-\lambda_{0}\right| \leq \Omega & \lambda_{1} \leq \lambda \leq \lambda_{2} \\
N_{s} & \left|\lambda-\lambda_{0}\right|>\Omega & \lambda_{1} \leq \lambda \leq \lambda_{2}
\end{array},\right.
$$




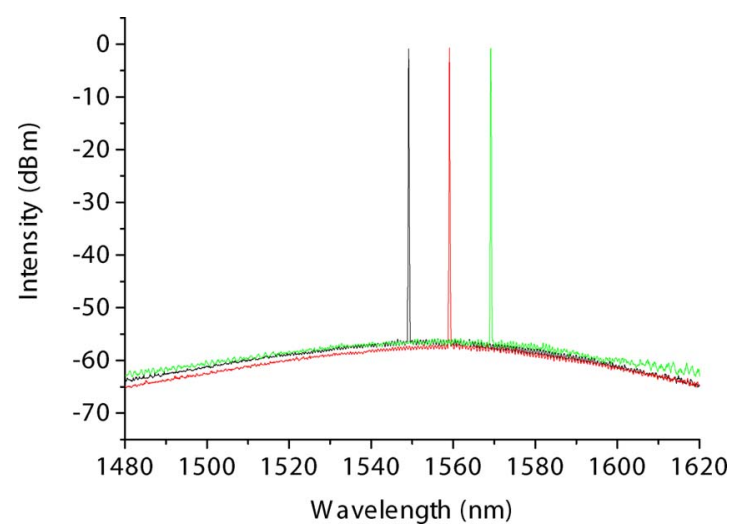

Fig. 2. (Color online) Measured spectral responses of a tunable laser.

where $P$ is the peak power spectral density, $\lambda_{0}$ and $\Delta \lambda_{0}$ are the center wavelength, and $-3 \mathrm{~dB}$ spectral width of the optical signal, respectively, and $\Omega$ is determined by the optical signal with a given level of noise which can be expressed as

$$
\exp \left[-4 \ln 2\left(\frac{\Omega}{\Delta \lambda_{0}}\right)^{2}\right]=\frac{N_{s}}{P}
$$
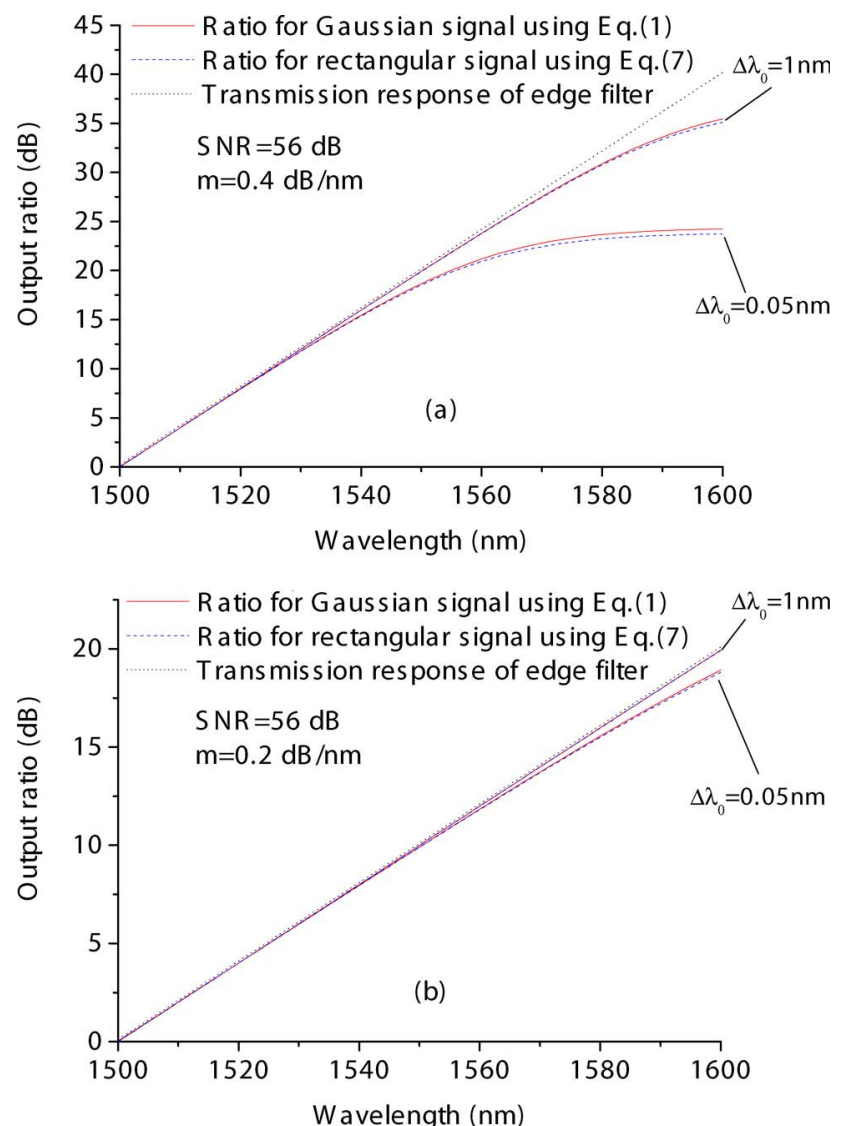

Fig. 3. (Color online) Transmission response of edge filters and corresponding ratios for input optical signals with different $\Delta \lambda_{0}$ when the slope of the edge filter $m$ equals (a) $0.4 \mathrm{~dB} / \mathrm{nm}$ and (b) $0.2 \mathrm{~dB} / \mathrm{nm}$.

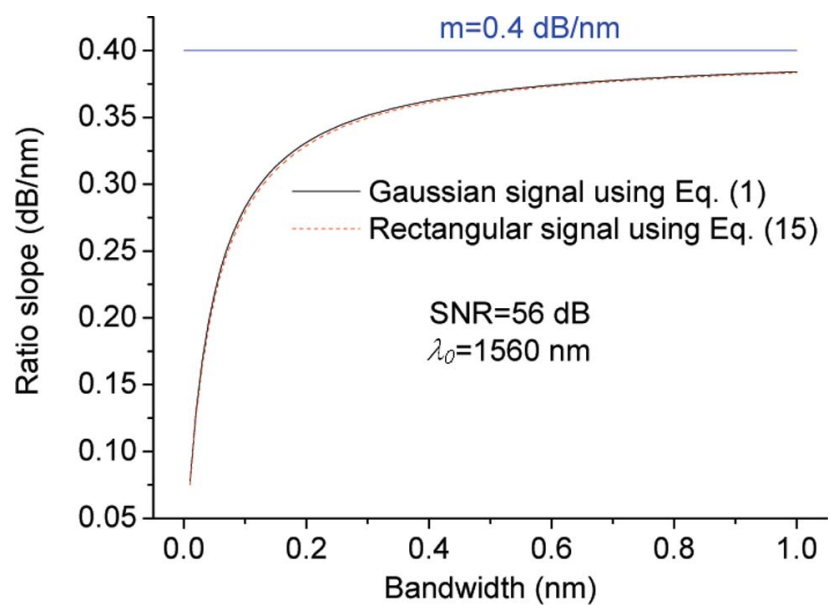

Fig. 4. (Color online) Ratio slope response variations versus optical bandwidth at $1560 \mathrm{~nm}$.

In our simulations, we used parameters of a real tunable laser source radiating in a wavelength range from 1480 to $1620 \mathrm{~nm}$. Several examples of the power spectral density spectra at different wavelengths are shown in Fig. 2. These spectra were measured with an optical spectrum analyzer (OSA), which has a resolution of $0.01 \mathrm{~nm}$, and the $-3 \mathrm{~dB}$ spectral width of the tunable laser was around $0.05 \mathrm{~nm}$, while the maximum output of the tunable laser was $0 \mathrm{dBm}$.

Simulations based on both rectangular and Gaussian signal spectra were carried out for comparison. Simulation results relating to the influence of bandwidth $\Delta \lambda_{0}$ are shown in Fig. 3 . In the simulation, we assume the input signal of the tunable laser has a $56 \mathrm{~dB}$ SNR over a wavelength range from 1480 to $1620 \mathrm{~nm}$. The peak power spectral density is $0 \mathrm{dBm} /$ $\mathrm{nm}$, and the inherent noise of both photodiodes is taken to be equivalent to a random optical input signal with a mean level of $-95 \mathrm{dBm}$ and $\mathrm{a} \pm 5 \mathrm{~dB}$ peak noise fluctuation. We assume zero insertion loss for the edge filter at $1500 \mathrm{~nm}$.

In the first instance, Fig. 3 shows that there is no significant difference between the ratio calculated for a rectangular signal spectrum with Eq. (7) and a Gaussian signal spectrum with Eq. (1). This indicates that the theoretical analysis above is still applicable to a Gaussian signal spectrum.

Figure 3 also shows that for a given SNR, the larger the $-\overline{3} \mathrm{~dB}$ bandwidth $\Delta \lambda_{0}$, the closer the ratio $R\left(\lambda_{0}\right)$ is to $\bar{T}\left(\lambda_{0}\right)$, and the larger the slope $S\left(\lambda_{0}\right)$ especially at a longer wavelength. The influence of the signal bandwidth is more significant when the slope of the edge filter is high, as shown in Fig. 3(a) with a $40 \mathrm{~dB}$ discrimination from $1500 \mathrm{~nm}$ to $1600 \mathrm{~nm}$.

Table 1. Signal-to-Noise Ratio and $3 \mathrm{~dB}$ Bandwidth Values for the Two Optical Sources

\begin{tabular}{lcc}
\hline Optical Sources & SNR & $3 \mathrm{~dB}$ Bandwidth $(\mathrm{nm})$ \\
\hline Tunable laser & 56 & 0.05 \\
FBG & 40 & $0.4-0.8$ \\
\hline
\end{tabular}




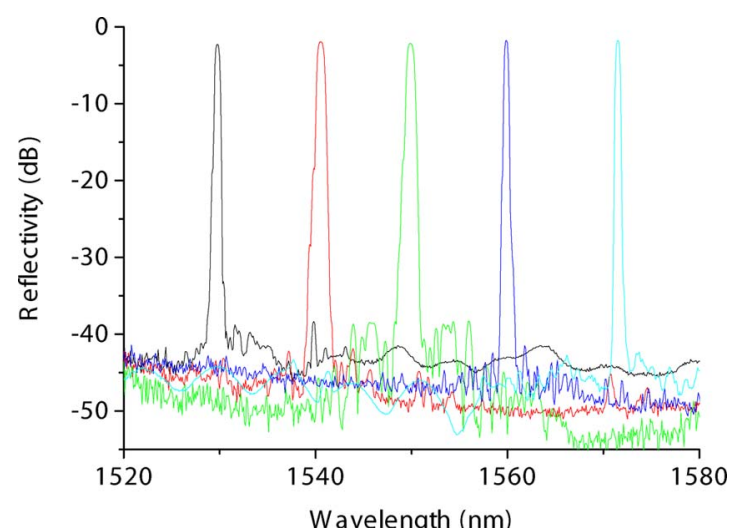

Fig. 5. (Color online) Measured spectral responses of five FBGs.

Figure 4 gives simulation results for the ratio slope response variations versus optical bandwidth of the input optical signal at a fixed wavelength. In this simulation, the parameters used are the same as above, and the wavelength selected is $1560 \mathrm{~nm}$.

Figure 4 shows that for both the Gaussian and rectangular signal, as the optical signal bandwidth increases, the slope and, hence, the resolution of the system increases. This result indicates that the ratiometric system will have a higher resolution when measuring the wavelength of an optical signal with a greater bandwidth. The simulation results agree well with the theoretical analysis above.

\section{Experimental Investigation}

The influence of the $3 \mathrm{~dB}$ bandwidth of an optical signal was investigated by measuring the ratio response using two types of optical sources. The two types of optical sources are (i) a tunable laser and (ii) five FBGs with center wavelengths at approximately $1530,1540,1550,1560$, and $1571 \mathrm{~nm}$ driven by a broadband optical source. The output signal of the tunable laser has noise distribution around a center wavelength $\lambda_{0}$ over a fixed range from $1480 \mathrm{~nm}$ to $1620 \mathrm{~nm}$, as shown in Fig. 2. The FBGs have a $-3 \mathrm{~dB}$ bandwidth $0.4-0.8 \mathrm{~nm}$. The $3 \mathrm{~dB}$ bandwidths and SNR values for the two optical sources are listed

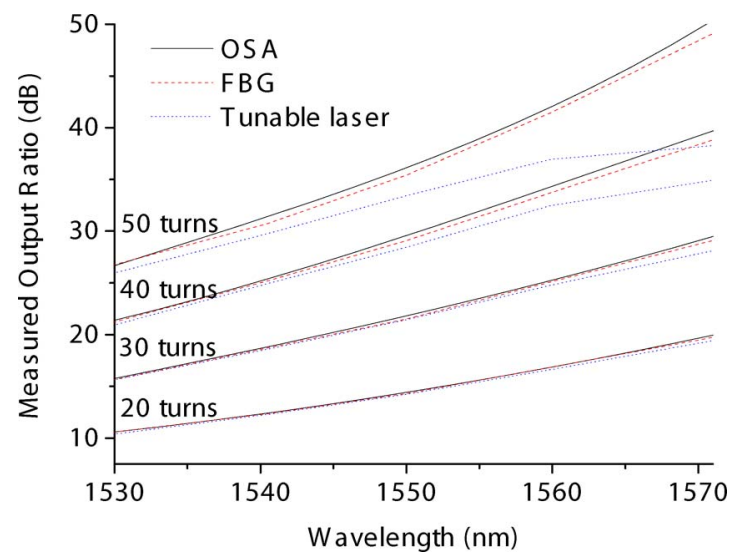

Fig. 6. (Color online) Measured transmission responses of the edge filters and corresponding system ratios. in Table 1, and the measured spectral response of the FBGs using an OSA is shown in Fig. 5.

Because of the fact that the five FBGs have center wavelengths of $1530,1540,1550,1560$, and $1571 \mathrm{~nm}$, the experiments were carried out over a wavelength range from $1530 \mathrm{~nm}$ to $1571 \mathrm{~nm}$ only. Experimental results are shown in Fig. 6. The edge filters used in the experiments are macrobending single-mode fiber SMF28 filters [15] with bending diameters of $21 \mathrm{~mm}$ and $50,40,3 \overline{0}$, and 20 turns (corresponding to $m=0.59,0.44,0.34$, and $0.23 \mathrm{~dB} / \mathrm{nm}$ ), respectively.

Figure 6 shows that when the slope of the edge filter is small (for example, filters with 20 and 30 turns), the ratio responses of the system for both types of the optical signal are both very close to the transmission response of the edge filter measured with OSA. However, when a tunable laser is used as an input optical source, as the slope of the edge filter increases (40 and 50 turns), the deviation of the measured ratio from the transmission response of the edge filter becomes significant, especially at longer wavelengths. On the other hand, the ratios measured for the FBG signals are matched well with the transmission response of the edge filter measured by an OSA. This can be explained as follows: although the tunable laser signal has a very high SNR (56 dB), its $-3 \mathrm{~dB}$ bandwidth is only $0.05 \mathrm{~nm}$, which means that the contribution to the detected power of the signal [corresponding to $A-A N_{s} / P$ in Eq. (7)] is small compared to the contribution of the noise [corresponding to $B N_{s} / P$ in Eq. (7)], especially at longer wavelengths; reflected signals from FBGs have a larger $-3 \mathrm{~dB}$ bandwidth of 0.4-0.8 nm but a lower SNR compared to that of the tunable laser-this makes the value of $A-A N_{s} /$ $P$ much greater than $B N_{s} / P$, even at longer wavelengths. As analyzed above, the larger the bandwidth, the greater the value of $A-A N_{s} / P$ compared to $B N_{s} / P$, the smaller the deviations of $R\left(\lambda_{0}\right)$ from $\bar{T}\left(\lambda_{0}\right)$, and thus the higher the slope $S\left(\lambda_{0}\right)$.

\section{Conclusions}

The bandwidth of an input optical signal influences significantly the resolution of a RWM system. In this paper, a theoretical analysis and numerical simulations of the influence of the optical signal bandwidth on the resolution of a ratiometric system are provided, supported by experimental verification. The conclusions are as follows: (i) if the noise of the photodiodes can be neglected and the SNR of the optical signal whose wavelength is being measured is high enough to be considered as infinite, the bandwidth of the input optical signal has no influence on the ratio slope $S\left(\lambda_{0}\right)$ of the ratiometric measurement system, which in this case is identical to the slope of the edge filter used. Therefore, the resolution of the ratiometric system is defined by the slope of the edge filter. (ii) If the noise of either the photodiodes or the input optical signal is significant, the higher the value of the optical signal bandwidth, the higher the ratio slope $S\left(\lambda_{0}\right)$, and, therefore, the higher the measurement resolution. 
In real-world wavelength measurement applications where optical signals have limited SNR, frequently the bandwidth of the input optical signal is unknown, leading to degradation of the resolution and accuracy of the wavelength measurements, when a RWM system is used However, in some situations, such as for the interrogation of FBG optical sensors, measurement resolution can be improved with RWM if both the SNR and bandwidth of the input optical signal are well known, because it is then possible to customize the RWM system to achieve better resolution and accuracy.

The authors acknowledge the support of Science Foundation Ireland under grant 07/SK/I1200.

\section{References}

1. S. M. Melle, K. Liu, and R. M. Measures, "A passive wavelength demodulation system for guided-wave Bragg grating sensors," IEEE Photon. Technol. Lett. 4, 516-518 (1992).

2. M. G. Xu, H. Geiger, and J. P. Dakin, "Modeling and performance analysis of a fiber Bragg grating interrogation system using an acousto-optic tunable filter," J. Lightwave Technol. 14, 391-396 (1996).

3. J. Mora, J. Luis Cruz, M. V. Andres, and R. Duchowica, "Simple high-resolution wavelength monitor based on a fiber Bragg grating," Appl. Opt. 43, 744-749 (2004).

4. Q. Wu, P. L. Chu, and H. P. Chan, "General design approach to multi-channel fiber Bragg grating," J. Lightwave Technol. 24, 4433 (2006).

5. Y. S. Hsu, L. K. Wang, W. F. Liu, and Y. J. Chiang, "Temperature compensation of optical fiber Bragg grating pressure sensor," IEEE Photon. Technol. Lett. 18, 874-876 (2006).
6. C. L. Zhao, M. S. Demokan, W. Jin, and L. Xiao, "A cheap and practical FBG temperature sensor utilizing a long-period grating in a photonic crystal fiber," Opt. Commun. 276, 242-245 (2007).

7. D. Grobnic, S. J. Mihailov, C. W. Smelser, and R. B. Walker, "Multiparameter sensor based on single high-order fiber Bragg grating made with IR-femtosecond radiation in single-mode fibers," IEEE Sens. J. 8, 1223-1228 (2008).

8. M. A. Davis and A. D. Kersey, "All fiber Bragg grating sensor demodulation technique using a wavelength division coupler," Electron. Lett. 30, 75-77 (1994).

9. Q. Wu, A. M. Hatta, Y. Semenova, and G. Farrell, "Use of a SMS fiber filter for interrogating FBG strain sensors with dynamic temperature compensation," Appl. Opt. 48, 54515458 (2009).

10. Q. Wu, P. Wang, Y. Semenova, and G. Farrell, "Influence of system configuration on a ratiometric wavelength measurement system," Meas. Sci. Technol. 21, 094013 (2010).

11. X. F. Yang, C. L. Zhao, Q. Z. Peng, Z. Q. Zhou, and C. Lu, "FBG sensor interrogation with high temperature insensitivity by using a HiBi-PCF Sagnac loop filter," Opt. Commun. 250, 63-68 (2005).

12. Q. Wang, G. Farrell, and T. Freir, "Study of transmission response of edge filters employed in wavelength measurements," Appl. Opt. 44, 7789-7792 (2005).

13. Q. Wu, G. Rajan, P. Wang, Y. Semenova, and G. Farrell, "Optimum design for maximum wavelength resolution for an edge filter based ratiometric system," Opt. Laser Technol. 42, 1032-1037 (2010).

14. A. T. Georges and S. N. Dixit, "Laser line-shape effects in resonance fluorescence," Phys. Rev. A 23, 2580-2593 (1981).

15. Q. Wang, G. Farrell, and T. Freir, "Theoretical and experimental investigations of macrobend losses for standard single mode fibers," Opt. Express 13, 4476-4484 (2005). 